\title{
Etnografía feminista en contextos de múltiples violencias*
}

\author{
Feminist ethnography \\ in contexts of multiple violences
}

\author{
R. AÍDA HERNÁNDEZ CASTILLO**
}

\begin{abstract}
In this article I will address the implications of making a feminist ethnography from collaborative methodologies. These collaborative methodologies recognize the privilege, but also the vulnerabilities, that we have as anthropologist-activists in social contexts marked by patriarchal violence. Taking as case studies three activist-research experiences with indigenous women victims of sexual violence, women in prison, and relatives of the disappeared persons, I reflect on the methodological strategies that can be used to make a feminist ethnography from an ethic of care and a decolonial perspective.
\end{abstract}

Key words: feminist research, self-reflection, collaborative methodology, emotional academic communities, extreme violence

\section{Resumen}

En este artículo se abordarán las implicaciones de hacer una etnografía feminista desde metodologías colaborativas, que reconocen los privilegios, pero también las vulnerabilidades que tienen las antropólogas-activistas, en contextos sociales marcados por las violencias patriarcales. Tomando como estudios de caso tres experiencias de investigación activista-con mujeres indígenas víctimas de violencia sexual, con mujeres en reclusión y confamiliares de personas desaparecidas-se reflexiona sobre las estrategias metodológicas que pueden servir para hacer una etnografía feminista, desde una ética del cuidado y una perspectiva descolonial.

Palabras clave: investigación feminista, autorreflexividad, metodologías colaborativas, comunidades emocionales académicas, violencias extremas

\section{Introducción: diálogos con la antropología fugitiva}

$\mathrm{E}$ n septiembre de 2016, durante mi estancia como profesora visitante en la Universidad de Texas en Austin, un grupo de estudiantes doctorales de antropología se acercó a mí para compartir un texto colectivo que escribían sobre sus experiencias de violencias y hostigamiento sexual durante el trabajo de campo. Todas ellas eran jóvenes consideradas "mujeres de color" en el contexto de Estados Unidos, quienes habían realizado trabajo de campo en Palestina, Guyana, Cuba, El Salvador y México (véase Berry et al., 2017). Su texto era una crítica a la visión institucionalizada del trabajo de campo como un rito de paso masculinista, reproducida aún por quienes reivindican una perspectiva crítica ante la academia positivista. Desde estas perspectivas,

\footnotetext{
* Artículo recibido el 25/03/21 y aceptado el 25/05/21.

** Centro de Investigaciones y Estudios Superiores en Antropología Social-CDMX. Juárez 87, col. Tlalpan Centro, 14000 Ciudad de México <aidaher2005@gmail.com>. oRcID: https://orcid.org/0000-0003-1066-9173
} 
las estrategias metodológicas de la investigación activista-que incluyen comprometerse en las luchas por la justicia y "poner el cuerpo en el campo"-son poco problematizadas, asumiendo un sujeto masculino que no enfrenta riesgos, ni vulnerabilidades al construir alianzas con los actores sociales con quienes trabaja. Su reclamo iba dirigido sobre todo a algunos de sus maestros, quienes las formaron dentro de la tradición de investigación activista (Gordon, 2007; Hale, 2008; Speed, 2008). Sus reflexiones aludían a la imagen de un sujeto radical idealizado subliminalmente masculino, que reproducía los silenciamientos de la antropología positivista, al no asumir el lugar de privilegio desde donde se hacía activismo e investigación.

Este señalamiento había sido hecho por Nancy Scheper-Hughes hace casi 40 años, cuando advirtió que el trabajo de campo se sustentaba en una mística casi machista, que llamaba a resistir con estoicismo los retos que se enfrentan durante la investigación (Scheper-Hughes, 1983). Si bien las antropólogas feministas han reflexionado de manera abierta sobre los dilemas éticos en el trabajo de campo (véase Wolf, 1996), pocas veces se ha escrito con claridad sobre las violencias que como mujeres, marcadas por múltiples exclusiones, podemos experimentar en él. Yo, en lo personal, me sentí interpelada por el reclamo, pues en mis escritos metodológicos sobre la investigación activista nunca había abordado mis propias experiencias de violencia en el campo (véanse Hernández Castillo, 2015, 2016a, 2016b). En cierta medida, mi labor con organizaciones indígenas y de migrantes partía de la premisa de que sus integrantes eran aliados políticos con quienes habría que construir relaciones de colaboración, reconociendo mi posición de privilegio étnica y de clase, en una sociedad profundamente racista y clasista como la mexicana, pero sin tematizar cómo mi identidad de género me ubicaba en contextos de vulnerabilidad.

Al leer sobre la agresión sexual sufrida por Maya Berry, en Cuba; las experiencias de hostigamiento sexual vividas por Elizabeth Velásquez Estrada, por parte de los jóvenes pandilleros con quienes trabajó en El Salvador; o el ambiente de terror en el que Sarah Ihmoud realizó su trabajo de campo en Palestina, estando embarazada y siendo intimidada por hombres armados judíos (véase Berry et al., 2017); me recordaron mis propias experiencias y temores al trabajar con migrantes magrebíes y senegaleses en el sur de España en 1993.

Me encontraba en el segundo año de mi doctorado en la Universidad de Stanford, cuando junto con mi amiga Liliana Suárez-Navaz conseguimos una beca para realizar trabajo de campo preliminar entre mi- grantes norafricanos y senegaleses en Andalucía. Alentada por mi maestro Rodolfo Stavenhagen, quien cuestionaba lo que llamaba el nacionalismo metodológico de los antropólogos mexicanos, decidí hacer mi tesis doctoral fuera de México. Convencí a Liliana para elaborar una tesis conjunta sobre los cambios de las políticas migratorias en España a partir de su incorporación a la Unión Europea y su impacto en la vida de los migrantes. Nos preparamos haciendo las lecturas obligatorias acerca de la migración africana en Europa, yo retomando mis clases de árabe, y ella avanzando en sus clases de francés (asumiendo que algunos migrantes norafricanos hablarían ese idioma). Orientalismo, de Edward Said (1978), estuvo entre los textos fundamentales que nos ayudaron a leer con ojos críticos los escritos de feministas occidentales en torno al islam. No queríamos reproducir los prejuicios y estereotipos sobre los hombres musulmanes que algunas feministas del Primer Mundo habían contribuido a perpetuar.

Cuando llegamos al pueblo de Andalucía donde planeábamos llevar a cabo nuestro trabajo de campo, nos encontramos con una comunidad de jóvenes migrantes, sobre todo de Marruecos y Senegal, que se sorprendieron al ver llegar a dos mujeres jóvenes a las galeras habitadas sólo por jornaleros hombres. Mis orígenes eran un enigma para ellos: el fenotipo moreno y el árabe estándar rudimentario con el que intentaba comunicarme los hicieron pensar que era hija o descendiente de árabes migrantes. Mis experiencias previas trabajando con refugiados guatemaltecos en los campamentos de Chiapas no me habían preparado para un contexto marcado por lo que entonces yo veía como una "coquetería agresiva” por parte de los hombres migrantes. Entre las familias de refugiados guatemaltecos, como mujer soltera, era considerada menor de edad, y las mujeres me tomaron bajo su protección; nunca me sentí insegura, tal vez un poco infantilizada, pero nunca hostigada. En cambio, en este ambiente mayoritariamente masculino, me sentía en extremo incómoda, vulnerable, y a la vez culpable por mi incomodidad; creía que estaba reproduciendo el orientalismo hacia los hombres musulmanes, que tanto habíamos criticado en nuestro Seminario de Investigación Pre-Campo.

Esta situación hizo crisis una noche en Granada, cuando, después de múltiples intentos por conseguir una entrevista con integrantes de las organizaciones de vendedores ambulantes, uno de sus representantes nos citó para una entrevista colectiva en un complejo habitacional en los barrios marginales de la ciudad. Eran como las nueve de la noche, y a la cita llegaron unos diez integrantes de la organización, en la 
que confluían norafricanos y senegaleses. El ambiente era tenso, se reían entre ellos, hablando en wolof, en bereber o en un dialecto del árabe magrebí que me costaba entender, era evidente que nosotras éramos el tema de conversación. Empezaron a bromear en castellano, cuestionando nuestra "audacia" de andar solas por el mundo, preguntando por nuestros novios o maridos y lanzándonos piropos, que a mí, en lo personal, me incomodaban muchísimo. Fue en un momento de silencio cuando tomé conciencia del riesgo en el que estábamos: dos mujeres solas, sin redes ni familia en la ciudad, en medio de la noche, rodeadas por desconocidos, quienes se burlaban de nosotras y tenían más interés en nuestra vida amorosa que en las preguntas laborales que les hacíamos. Así, me di cuenta de que no podía trabajar con personas hacia las que no sentía empatía y con quienes me sentía vulnerable.

En ese entonces reflexioné mucho sobre la diferencia que veía entre mi formación en la antropología crítica mexicana, donde la colaboración y el compromiso político con los colectivos con quienes trabajas resultaba fundamental, y una formación anglosajona, donde el problema teórico era lo central y después se decidía si la investigación se efectuaba en Guatemala o en Tortola. Si no estaba dispuesta a hacer un compromiso de largo aliento con las organizaciones de migrantes, creía que era antiético realizar una tesis doctoral que sólo sirviera para mi titulación. Mi corazón tenía que estar donde estaban mis inquietudes académicas y políticas, y esa noche en Granada me resultó patente que no era en tierras andaluzas. Liliana SuárezNavaz, quien con el tiempo se convertiría en mi comadre y hermana, entendió y respetó mi decisión, y le dio continuidad al proyecto, que tuvo como producto una excelente tesis doctoral, publicada años después como libro (Suárez-Navaz, 2006).

Mi fallida experiencia de campo con los migrantes en la península ibérica y los miedos que viví en ese verano andaluz sólo los había compartido en charlas informales con amigas, nunca escribí sobre ello, ni profundicé sobre las implicaciones de ser una mujer joven tercermundista ("de color", dirían en Estados Unidos), trabajando en contextos masculinos. Inconscientemente, creí que reconocer mis miedos y vulnerabilidades era ir en contra del ethos antropológico en el que me había formado, donde los "verdaderos antropólogos" se adaptan a los entornos poco propicios y enfrentan los peligros con valentía.

Tuvieron que pasar casi 30 años para que pudiera reflexionar y escribir sobre el modo en que las jerarquías de género, edad y étnico-raciales marcaron mi experiencia de trabajo de campo en territorio europeo, influyendo en mis decisiones posteriores de trabajar preferentemente con colectivos de mujeres. La antropología fugitiva, a la cual nos invitan las jóvenes antropólogas egresadas de la Universidad de Texas en Austin, busca escapar del ethos masculino, en el que el riesgo es parte del rito de paso del trabajo de campo. Nos convocan a una ética del cuidado desde una praxis feminista, que reconoce privilegios y vulnerabilidades.

A lo largo de estos 27 años que han pasado, desde mis experiencias andaluzas, la vida me ha llevado a trabajar en contextos de múltiples violencias. No siempre como elección teórica y política, sino porque los contextos en los que habito y trabajo en México han estado en las últimas décadas caracterizados por un continuum de violencias. La antropología ciudadana que se ha desarrollado en América Latina ha significado hacer investigación activista sobre temas que nos competen como ciudadanos y en espacios e instituciones que muchas veces compartimos con las actoras y los actores sociales con quienes trabajamos (Jimeno, 2000).

En este artículo quisiera reflexionar en torno a las implicaciones de hacer una etnografía feminista desde metodologías colaborativas, que reconocen los privilegios, pero también las vulnerabilidades que tenemos las antropólogas-activistas en contextos sociales marcados por las violencias patriarcales.

\section{Investigando las violencias de género}

La cuestión de las violencias de género llegó a mi vida como tema de investigación de un modo inesperado cuando regresé a Chiapas a hacer mi tesis doctoral sobre las políticas estatales en la frontera MéxicoGuatemala, donde había participado en proyectos educativos desde mis estudios de licenciatura (véanse Hernández Castillo, 2001a y 2001b). Mis perspectivas acerca de la antropología del Estado se vinculaban con mis preocupaciones teórico-políticas sobre las violencias que el nacionalismo mexicano había infligido en las vidas de la población indígena del país. Las perspectivas feministas no eran entonces parte de mi marco teórico, y mi preocupación por el análisis sistémico y la violencia estatal me hacía perder de vista las violencias cotidianas que las mujeres vivían en los espacios comunitarios.

A partir de la violación sexual de mi compañera de casa, y de mi acompañamiento en su proceso de denuncia, atestigüé las violencias de género en los espacios de justicia, y me empecé a involucrar en un movimiento feminista contra la violencia de género (véase Hernández Castillo, 2016a). A principios de la década de los 
noventa se formó el Movimiento Amplio de Mujeres en Chiapas, con la confluencia de mujeres indígenas y mestizas, con distintas trayectorias organizativas, que se unieron en contra del autoritarismo y la violencia imperantes durante la gubernatura de Patrocinio González Garrido (1988-1993). Se trataba de un movimiento amplio que no se autodefinía como feminista; sólo un grupo minoritario dentro del mismo, sobre todo de académicas, tenía una formación feminista y vínculos estrechos con el movimiento feminista nacional. Fueron ellas las primeras en reivindicar el término feminista para el movimiento, reivindicación que hasta la fecha se toma con recelo por muchos sectores de las mujeres organizadas. En otros escritos he analizado las tensiones y los retos de este movimiento amplio (véase Hernández Castillo y Espinosa Damián, 2012). Basta decir aquí que las genealogías políticas eran múltiples y que la mayor o menor identificación con una agenda abiertamente feminista se fue construyendo poco a poco, la mayoría de las veces en paralelo a las luchas por la tierra, o por la autonomía indígena.

Fueron las mujeres indígenas y campesinas quienes nos forzaron a replantear nuestras perspectivas limitadas sobre la violencia de género, al señalar que el despojo territorial y la destrucción de sus bosques por las compañías madereras eran también violencias que se ejercían sobre las mujeres, que daban conti-

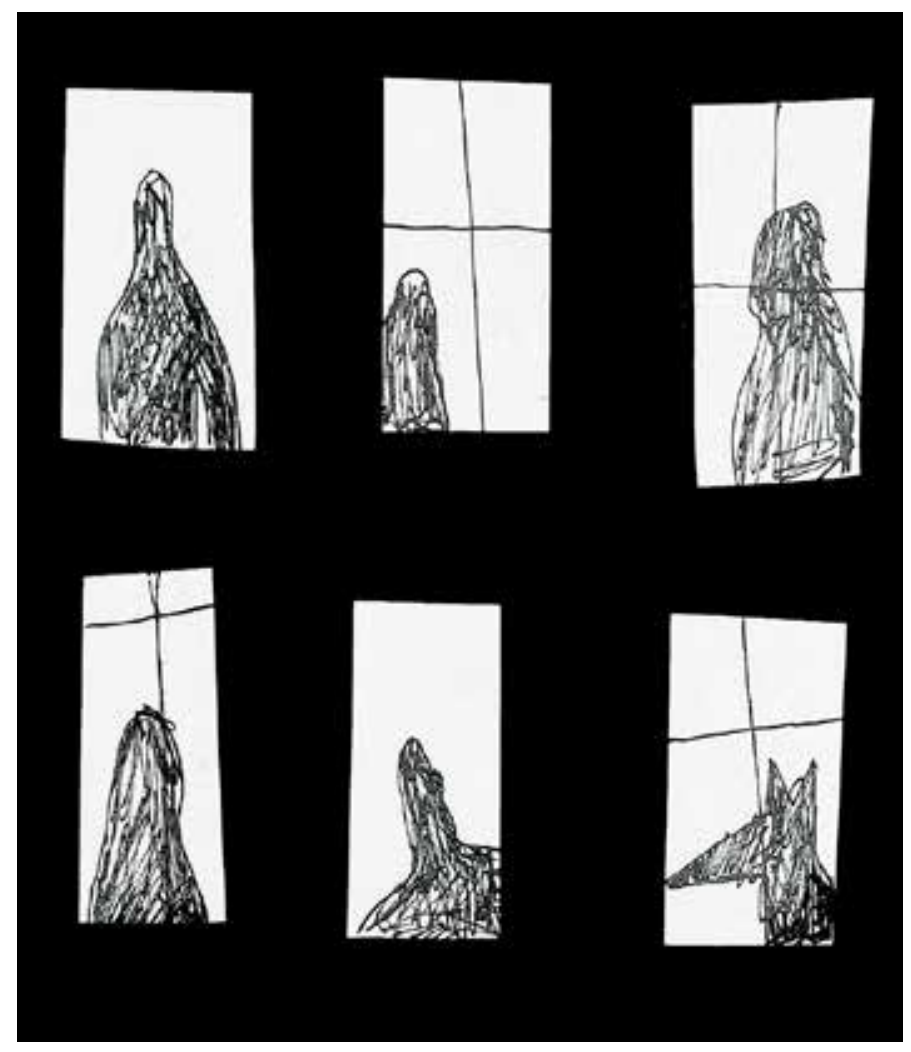

nuidad a un legado colonial de violencias que había afectado sus vidas. Estas teorizaciones, que muchas veces se exponían en forma de manifiestos políticos o relatorías de encuentros, me llevaron a poner en tela de juicio el etnocentrismo de nuestras agendas feministas y a buscar otras posibilidades de construir alianzas desde los diálogos de saberes (véase Hernández Castillo, 2001c).

En razón de estos diálogos con mujeres indígenas y campesinas, dentro de nuestra organización empezamos a cuestionar nuestras propias visiones del mundo y a relativizar nuestras verdades. El género, la autoestima, el empoderamiento, la subordinación y la resistencia se convirtieron en puntos de llegada y no de partida. Es decir, el reto metodológico y político consistía entonces en hacer a un lado nuestras preconcepciones dicotómicas sobre lo femenino y lo masculino; lo público y lo privado; el poder y la resistencia; para partir de un análisis conjunto de las realidades específicas que nos facilitara la construcción de nuevos conceptos que respondieran al contexto cultural en el que realizábamos nuestra labor.

Ésta ha sido desde entonces mi apuesta política y académica en lo que respecta a mi trabajo en relación con la violencia doméstica. Haciendo eco de las ideas de Chandra Mohanty, considero que: "La violencia masculina debe ser teorizada e interpretada dentro de sociedades específicas, para así poder entenderla mejor y poder organizarnos más efectivamente para combatirla" (Mohanty, 1984: 339). Si el reconocimiento de las similitudes entre las mujeres nos permite crear alianzas políticas, el reconocimiento de las diferencias ha sido un requisito indispensable para la construcción de un diálogo respetuoso así como para la búsqueda de estrategias de lucha más acordes con las distintas realidades culturales. La etnografía feminista ha sido una herramienta fundamental en la documentación y la comprensión de estas similitudes y diferencias.

\section{Genealogía de la etnografía feminista}

Mi conciencia y militancia feminista transformaron por completo mi modo de entender la antropología y de hacer investigación de campo. Mi mirada, centrada en el Estado y sus instituciones, empezó a percibir la importancia de los espacios domésticos y las violencias cotidianas, para documentar y entender la forma en que las violencias estructurales marcaban las vidas de las mujeres. Mis primeros acercamientos a la antropología feminista me mostraron el sesgo androcéntrico que tenían las etnografías clásicas y me llevaron a cuestionar el concepto esencialista de 
cultura que había excluido las voces y las experiencias de las mujeres, presentando los discursos hegemónicos en torno a la tradición y al "deber ser comunitario" como las voces que hablaban por las culturas de los pueblos (Moore, 2009).

En la academia angloparlante, esta crítica ha ido acompañada por una reflexión feminista sobre la experiencia misma del trabajo de campo, cuando el cuerpo y la subjetividad que se ponen en juego son los de una mujer antropóloga (véanse Bell, Caplan y Wazir, 1993; Wolf, 1996). En el mundo hispanoparlante, las nuevas generaciones de antropólogas han escrito y discurrido con amplitud sobre lo que implica poner el cuerpo y teorizar desde las emociones en contextos marcados por las violencias (véanse Ruiz Trejo, 2016; De Marinis, 2017; García-Santesmases Fernández, 2019; García González, 2019).

Esta literatura nos ha mostrado que hacer etnografía feminista supone ir más allá del empirismo que ha caracterizado a la llamada observación participante de la antropología tradicional, y que requiere reconstruir el sentido que tienen prácticas, discursos, reivindicaciones políticas, a partir de las concepciones culturales de los actores sociales con quienes se trabaja, reconociendo la manera en que las estructuras de género determinan esos significados. Las etnografías feministas, desde distintas perspectivas teóricas, han rechazado la pretensión de neutralidad de las ciencias sociales positivistas, y por ello han enfrentado el reto de atreverse a incidir en la transformación social, con todos los riesgos que esto conlleva (Reinharz y Davidman, 1992). La tentación de imponer nuestras propias perspectivas de justicia y emancipación, reproduciendo los legados coloniales que hemos heredado del humanismo cristiano, está siempre ahí. La advertencia hecha hace más de tres décadas por Chandra Mohanty (1984) sobre los peligros del colonialismo epistémico de los feminismos urbano-centrados sigue teniendo vigencia, pues la denuncia de la opresión de las mujeres pobres puede caer con facilidad en la revictimización. Esto es en extremo delicado cuando se trata de contextos de múltiples violencias, en los cuales la extracción del testimonio y su exposición en el debate público puede ser fácilmente una apropiación del sufrimiento ajeno, que contribuya a una pornografía de la violencia que alimentan los medios de comunicación (véase Kleinman y Kleinman, 1996).

La construcción de un sujeto sufriente (suffering subject) por parte de la antropología de las violencias ha sido cuestionada con amplitud por autores como Joel Robbins (2013). Este autor plantea que, ante las críticas a la antropología como herramienta colonial que contribuyó a la construcción de un imaginario exotizante alrededor de los salvajes, la disciplina ha vuelto su mirada al entorno propio del investigador o investigadora, pero concentrando su mirada analítica en las personas que sufren, como su nuevo objeto de estudio. Estas representaciones tienden a sobredimensionar las violencias, sin reconocer las capacidades de resiliencia y solidaridad humana, que muchas veces están presentes en los contextos de violencias extremas.

Para evitar contribuir a la industria de la extracción del testimonio y a la construcción del sujeto sufriente, es cardinal una reflexión crítica feminista en torno a la escritura y la representación del dolor. Las metodologías dialógicas y colaborativas hacen que el dilema ético acerca de la representación del sufrimiento humano no sea sólo un problema de la investigadora, sino un tema de reflexión política colectiva, en el que resultan fundamentales las voces, las experiencias y los deseos de las actoras sociales con quienes se trabaja.

Las metodologías dialógicas, si en verdad se desea descolonizar la etnografía feminista, implican cambiar las formas de hacer investigación por estrategias más colaborativas en las cuales el problema mismo de investigación se defina en diálogos con las actoras sociales con quienes trabajamos. Pero también se requiere de maneras más creativas y participativas de presentar los resultados, en las que el tema de la representación de las violencias y las resistencias a ellas sea discutido y decidido de forma dialógica.

Ha corrido mucha tinta desde que James Clifford afirmara en su ahora clásica antología Writing Culture (1986) que las antropólogas feministas no habían aportado nada a la escritura etnográfica sobre las culturas. La respuesta contundente a la posición machista y excluyente de Clifford, por parte de Ruth Behar y Deborah Gordon, produjo otro de los clásicos de la antropología feminista: Women, Writing Culture (1995). Por décadas, las feministas han escrito del sufrimiento humano desde estrategias textuales que cruzan las fronteras de los géneros literarios y teorizan desde el sentir-pensar (véanse Anzaldúa, 1987; Behar, 2003; hooks, 2000; Lorde, 1984). Una nueva generación de antropólogas feministas está usando la escritura creativa (Tamez, 2007; Velásquez Nimatuj, 2018), los recursos audiovisuales (Corral, 2016; Rivera, 2018) y el mundo digital, ${ }^{1}$ para denunciar la continuidad de la violencia patriarcal, y para reconocer la capacidad de resistencia de las mujeres en diversos contextos de violencias.

1 Luchadoras <https://luchadoras.mx/>. 
La desestabilización de lo que se entiende por escritura etnográfica es parte de las resistencias feministas ante un canon que no sólo había impuesto sus teorías y formas de hacer investigación, sino que había establecido el lenguaje de la teoría, excluyendo aquellas voces que se expresaban a través de otras estrategias textuales. Hacer una etnografía feminista de las violencias entraña atreverse a experimentar con el lenguaje escrito, visual y artístico, reconociendo siempre la creatividad y las formas de resistencia de las mujeres con quienes trabajamos y construimos alianzas.

\section{Alianzas y coproducción de conocimientos en contextos de violencias extremas}

En la última década, mi investigación activista en torno a la violencia de género ha conllevado nuevos retos al complejizarse las manifestaciones de la misma en contextos signados por las complicidades entre el crimen organizado y los representantes del Estado. A la violencia estructural y la violencia doméstica, que marca la vida de miles de mujeres en México, se ha aunado el impacto de violencias extremas, que han hecho del feminicidio y la desaparición forzada prácticas comunes entre actores armados que controlan distintas regiones del país.

Se habla de una de las peores crisis de derechos humanos en la historia nacional, que parece tener continuidad con el nuevo gobierno del presidente Andrés Manuel López Obrador (2018-2023). La numeralia del terror nos habla de 350000 asesinados en la "Guerra contra el narco" (2006-2021), 94419 desaparecidxs, 52000 cuerpos no identificados bajo custodia del Estado, 70000 migrantes en tránsito desaparecidos y 4806 fosas clandestinas; esto tomando en cuenta sólo los datos oficiales, que los organismos de derechos humanos han señalado como un subregistro. La desaparición de personas, la mutilación de los cuerpos y su ocultamiento en fosas clandestinas se han convertido en parte de una pedagogía del terror, que utiliza los cuerpos como símbolos para marcar territorios, mantener el terror y controlar a la población (véase Segato, 2013).

Este ambiente de violencia e impunidad trae aparejado nuevos retos metodológicos y epistemológicos para la etnografía feminista y para la antropología social en un sentido más amplio. En el nivel metodológico, el trabajo de campo de larga duración en regiones afec- tadas por la violencia trae consigo múltiples peligros para investigadoras y estudiantes. Esto nos obliga a buscar estrategias colectivas de investigación desde equipos interdisciplinarios que trabajen de manera colaborativa con organizaciones de la sociedad civil.

En la última parte de mi artículo quisiera compartir algunas reflexiones teóricas, metodológicas y ético-políticas sobre los aprendizajes y retos que he enfrentado para llevar a cabo una investigación feminista en el contexto de la crisis de derechos humanos que vivimos en México. Mis reflexiones surgen de tres experiencias de investigación activista que he venido desarrollando en la última década:

1. La primera se refiere a mi labor acompañando los procesos de mujeres indígenas víctimas de violencia militar y paramilitar en Chiapas $\mathrm{y}$ Guerrero. La elaboración de peritajes antropológicos para la Corte Interamericana de Derechos Humanos y para órganos de justicia nacional me han permitido acercarme a las formas específicas de entender el agravio y la justicia desde conceptos de persona que desestabilizan los puntos de vista del derecho liberal. La elaboración de los peritajes a través de metodologías dialógicas y la coproducción de conocimiento desde un feminismo que se pretende descolonial me han llevado a confrontar, aunque sea mínimamente, las jerarquías epistémicas que tienden a reificar el sistema judicial. Asimismo, entender como parte del agravio el entorno de violencias estructurales e impunidad que posibilitó el uso de la violencia sexual como forma de tortura, fue un aprendizaje fundamental de estas experiencias, que abordaré más adelante (véanse Hernández Castillo, 2016b, 2017). ${ }^{2}$

2. La segunda corresponde a mi labor durante 11 años con mujeres presas que han apostado por confrontar la violencia penitenciaria mediante la escritura. Ésta ha sido para mí una de las experiencias de investigación más transformadoras y duraderas que he tenido en mi carrera académica. El desarrollo de una investigación académica que documentó el racismo judicial a partir de las historias de vida y la revisión de expedientes judiciales de mujeres indígenas presas se ha convertido en un proyecto de vida acompañando a la primera editorial penitenciaria del país, la Colectiva Hermanas en la Sombra,

\footnotetext{
2 Mi incursión en los peritajes antropológicos la hice con Héctor Ortiz Elizondo, quien ha sido mi coautor en varios informes periciales y textos académicos y mi amigo entrañable por más de 30 años.
} 
que a la fecha ha publicado 17 libros. ${ }^{3}$ Las mujeres presas con las que he trabajado vivieron múltiples violencias antes, durante y después de su detención, y en la presente administración habitan cárceles que se encuentran bajo el control de los carteles de las drogas. Sus libros de cuento, poesía, narrativa son formas creativas de teorizar sobre la intersección de violencias que han marcado sus vidas, y tienen mucho que enseñarnos acerca de los vínculos entre la violencia patriarcal, el racismo y las múltiples violencias del despojo y la desposesión capitalista (véanse Hernández Castillo, 2013, 2017, 2021).

3. La tercera es la investigación con familiares de personas desaparecidas, como parte del Grupo de Investigación en Antropología Social y Forense (GIASF) y posteriormente del Laboratorio de Antropología Jurídica y Forense del Centro de Investigaciones y Estudios Superiores en Antropología Social (CIESAS). Mi labor con madres y esposas de desaparecidos y desaparecidas de Sinaloa y Morelos me ha acercado a lo que Rita Laura Segato (2013) ha denominado la pedagogía del terror, en su forma más cruda, al acompañar procesos de exhumación de hijos, hijas, esposos o padres. Si bien nuestra investigación colaborativa como GIASF con estos colectivos podría ubicarse dentro de lo que algunos autores han llamado el giro forense 4 en la antropología, el contexto en el cual se realizan estas exhumaciones difiere sustancialmente de lo descrito por la creciente literatura en torno a lo que Francisco Ferrándiz ha nombrado etnografías a pie de fosa (véanse Ferrándiz, 2014; Sanford, 2003; Rojas-Perez, 2017). No se trata de un contexto de justicia transicional en el que las exhumaciones puedan ser utilizadas para procesos jurídicos de graves violaciones a los derechos humanos, como nos refieren Victoria Sanford para el caso guatemalteco, Juan Pablo Aranguren para el colombiano (2016) o Isaias Rojas-Perez para el peruano. ${ }^{5}$ Se trata de exhumaciones hechas por las propias madres, que no buscan encontrar a los culpables o judicializar los casos, como ellas mismas señalan; su objetivo es "encontrar a sus tesoros y darles una sepultura digna”. Esto no lo llevan a cabo en el marco de una escenografía científica forense, ni con una amplia cobertura mediática -como nos detallan desde una etnografía densa los trabajos antes citados-, sino en el de contextos de violencia, donde los perpetradores siguen actuando con libertad y muchas veces continúan ocupando puestos como servidores públicos en los organismos de seguridad locales. En estos entornos, hacer investigación colaborativa es poner el cuerpo en el centro de la violencia (véanse Hernández Castillo, 2019a; Hernández Castillo y Robledo Silvestre, 2020).

No es mi intención en este texto abordar en profundidad estas tres experiencias, pero sí quiero compartir algunos de los aprendizajes y retos que ha supuesto realizar investigación desde una perspectiva feminista dialógica y descolonial, en contextos marcados por violencias extremas.

\section{Las complejidades de las violencias patriarcales}

En los tres escenarios etnográficos en donde he venido desarrollando mis investigaciones en esta última década, las fronteras entre las fuerzas de seguridad $\mathrm{y}$ los integrantes del crimen organizado son muy difusas. A diferencia de los procesos de represión política de las dictaduras latinoamericanas, donde los principales perpetradores eran fuerzas de seguridad o grupos paramilitares vinculados con el Estado, en México estamos ante distintos tipos de perpetradores que actúan con diferentes estrategias de violencia y control territorial dependiendo del contexto local. En muchas regiones, los agentes de la violencia son fuerzas de seguridad, municipales, estatales, federales, o fuerzas castrenses, relacionadas con el crimen organizado; en otras, las organizaciones criminales actúan de manera directa en ambientes de impunidad. Pero lo que sí está claro es que, en 99 por ciento de los casos, los perpetradores son hombres violentos.

3 Se trata del proyecto colectivo Globalización, Justicia y Derechos desde una Perspectiva de Género y Poder, coordinado conjuntamente con María Teresa Sierra y cuyos resultados sistematizamos en el libro Justicias indígenas y Estado. Violencias contemporáneas (Sierra Camacho, Hernández Castillo y Rachel Sieder, 2013).

4 El término en inglés que se ha popularizado es el de forensic turn (véanse Ferrándiz, 2014; Dreyfus y Anstett, 2015).

5 El concepto de justicia transicional ha sido puesto en el centro del debate político por el gobierno de centro-izquierda de Andrés Manuel López Obrador. Esta apropiación ha sido muy cuestionada, porque se trata de un concepto que está siendo impuesto desde arriba y sin considerar las características específicas de México. Las condiciones de verdad, justicia, reparación y no repetición no se cumplen en un entorno en el que el crimen organizado sigue controlando regiones enteras del país. 
Los militares que violaron a Inés Fernández Ortega y Valentina Rosendo Cantú, dirigentes del pueblo me'phaa en la montaña de Guerrero; los policías que detuvieron y torturaron sexualmente a muchas de las mujeres presas en Atlacholoaya, Morelos; y aquellos que asesinaron y tiraron en fosas clandestinas los cuerpos que ahora son exhumados por Las Rastreadoras de El Fuerte, Sinaloa; todos ellos compartían culturas masculinas violentas, en entornos donde la impunidad da permiso para matar.

Es decir, una de las raíces de la crisis de derechos humanos que se vive en México está en la violencia patriarcal, un problema sobre el cual las feministas hemos trabajado históricamente. Sin embargo, en la academia mexicana, el análisis y el activismo feministas se han centrado sobre todo en el tema del feminicidio, por su urgencia y gravedad. El análisis feminista de la desaparición forzada, del desplazamiento y de las masacres de indígenas, campesinos y migrantes, son aún cuestiones pendientes, como lo son las alianzas entre las organizaciones feministas mexicanas y los familiares de desaparecidos y masacrados. En el interior de un feminismo radical y autónomo existe la postura de que se trata de un problema de "hombres que se matan entre ellos" y, por lo tanto, no le compete al feminismo.

Desde una postura interseccional y antirracista, varias de las integrantes de la Red de Feminismos Descoloniales, de la que soy parte, hemos señalado que son hombres y mujeres pobres y racializados las principales víctimas de la violencia patriarcal del Estado y de los grupos criminales. ${ }^{6}$ Esta violencia patriarcal se ejerce sobre cuerpos construidos como desechables por un sistema neocolonial, clasista y racializado, que hace posible estas formas extremas de violencia. Es decir, el racismo deshumaniza y vuelve ininteligible el valor de los cuerpos ubicados en territorios racializados (Cacho, 2012; Hernández Castillo, 2019b).

En paralelo, las masculinidades violentas promovidas por el sistema capitalista y patriarcal han encontrado un nicho de mercado en el crimen organizado, donde su capacidad de violencia está bien cotizada. Este capitalismo gore, como ha sido definido por la crítica feminista Sayak Valencia (2010), ha profundizado las exclusiones de la sociedad mexicana, dejando a un amplio sector de los hombres pobres y racializados sin posibilidades de cumplir el papel de proveedores que les asigna el sistema patriarcal. Frente a una cultura capitalista de consumo, que a la vez que deshumaniza promueve valores que decretan que, para "ser alguien" en el mundo, tienes que poder comprar un carro, un iPhone, un iPad, estos jóvenes han encontrado en su capacidad de violencia una mercancía cuya venta les da poder y control sobre cuerpos y territorios.

Un análisis y una praxis feministas descoloniales tendrían mucho que aportar a la construcción de una justicia transformadora, fuera de las estrategias y los marcos punitivos que no reconocen las múltiples intersecciones de violencias y desigualdades que dejan una impronta en la vida de víctimas y perpetradores, y que vuelven muy difusas las fronteras que separan a unos y otros. La desaparición de jóvenes pobres y racializados, o su asesinato como parte de las violencias del crimen organizado, deja secuelas indelebles en la vida de sus madres, sus esposas, sus hermanas (véase Smith, 2016). No se trata sólo de "hombres matándose entre ellos", sino de una violencia patriarcal que afecta a familias y comunidades enteras, sobre todo en regiones pobres y racializadas.

Ante esta compleja realidad, han sido las mujeres organizadas desde el interior de las prisiones, en organizaciones indígenas, o en colectivos de familiares desaparecidos, quienes nos han dado algunas pistas de cómo promover estrategias de reconstrucción del tejido social y cómo repensar la justicia desde otros marcos y epistemologías, que no siempre pasan por la justicia del Estado.

\section{Pistas metodológicas para una etnografía feminista de las violencias}

No es mi propósito desarrollar en este artículo los resultados de estas investigaciones, que se han fundamentado en metodologías colaborativas descoloniales y que han acompañado diversas experiencias de activismo legal. El proceso mismo de las investigaciones ha sido tan relevante como la producción final, pues nos ha permitido acompañar la creación de espacios de reflexión colectiva, que han sido también espacios de formación para los equipos de investiga-

\footnotetext{
6 Retomo el concepto de interseccionalidad desarrollado por Kimberlé Crenshaw (1991 [1989]) para dar cuenta del carácter imbricado de las relaciones de poder de raza, clase y género que marca la vida de las mujeres pobres y racializadas. No obstante, por el contexto específico en el que surge la propuesta, no se exploraba la dimensión epistemológica, ni las construcciones de sentido en torno a la persona, la justicia y el resarcimiento de las mujeres cuyas vidas eran marcadas por esas intersecciones de violencias. El concepto de interseccionalidad permite dar cuenta de la manera imbricada en que funcionan los sistemas de opresión, pero resulta limitado para entender, desde contextos indígenas, otras formas de ser y estar en el mundo, que parten de sentidos de persona que van más allá del cuerpo físico.
} 
ción, a través de diálogos de saberes. Paralelamente a los productos académicos, he podido contribuir con la elaboración de peritajes antropológicos, bases de datos, productos de divulgación como programas de radio, documentales, manuales y libros gráficos, con el propósito de aportar a las estrategias de denuncia o al fortalecimiento de las estrategias de lucha contra las violencias y por la justicia. ${ }^{7}$

Compartiré a continuación por lo menos tres de las enseñanzas que estas experiencias de investigación activista y diálogos de saberes me han dejado y que considero esenciales para quienes realizan investigación en contextos de múltiples violencias.

\section{La construcción de comunidades de conocimiento $y$ emocionales dentro de la academia}

La complejidad de los retos que enfrentamos en estos contextos de violencias estructurales, cotidianas y extremas, vuelve necesario un enfoque interdisciplinario que posibilite entender la manera en que los procesos económicos globales han incidido no sólo en los mercados ilícitos de drogas, personas y mercancías, sino también en la construcción de identidades violentas y deshumanizadas, como los sujetos endriagos descritos por Sayak Valencia (2010). La geografía crítica nos permitiría ubicar estos procesos en el espacio, documentando la distribución de estas violencias en geografías racializadas y analizando los vínculos entre cuerpo y territorio, que las feministas han venido señalando (Belausteguigoitia Rius y Saldaña-Portillo, 2015).

La historia de la represión y la impunidad en México, y de la relación entre el Estado y el crimen organizado, nos ayudará a entender el continuum de violencias que apuntan los distintos testimonios de las víctimas, y a desentramar las complejidades de los poderes fácticos que ejercen y hacen posible las violencias.

Acercarnos a la cotidianidad de estas violencias, desde una etnografía feminista densa que ponga en el centro las experiencias de los actores y actoras con los que trabajamos, permitirá ver las formas específicas que toman los procesos globales descritos por los análisis sistémicos. Tejer fino a partir de estas distintas perspectivas aporta a una visión más compleja de los procesos y, además, producir conocimiento en colectivo nos ayuda también a compartir nuestros miedos e incertidumbres y construir comunidad en contextos en los que la competencia promovida por la academia neoliberal nos ha aislado cada día más. Como investigadoras, no somos inmunes a las violencias que afectan las vidas y los territorios de las actoras sociales con quienes trabajamos, y el solo peligro latente es ya una forma de violencia que aqueja nuestras mentes y cuerpos. En vez de silenciar estas afectaciones, como lo hace el ethos masculinista del trabajo de campo cuestionado por Maya Berry y sus colegas (2017), es importante reconocer nuestros miedos, socializarlos y gestionarlos de manera colectiva. Buscar estrategias de seguridad y autocuidado que nos permitan, en la medida de nuestras posibilidades, protegernos y proteger a las personas con quienes trabajamos. La construcción de comunidades emocionales, descritas por Myriam Jimeno (2010) y reivindicadas teórica y políticamente por muchas feministas latinoamericanas (Macleod y De Marinis, 2019), implica un ejercicio de autocuidado, sororidad y empatía, que nos ayuda a resistir colectivamente las afectaciones cotidianas que las violencias dejan en nuestros cuerpos y mentes.

\section{La representación y la sanación ante la violencia, el dolor propio y ajeno}

Retomando los llamados de atención que nos hace Veena Das (2008) a través de su antropología del dolor, es cardinal profundizar sobre los dilemas que enfrentamos cuando las estructuras conceptuales de nuestras disciplinas traducen el sufrimiento en un lenguaje distinto que les quita la voz a las víctimas $\mathrm{y}$ nos distancia de la inmediatez de su vivencia. Yo he intentado en mis escritos dar cuenta de este dolor reproduciendo con amplitud sus propios escritos, pero sobre todo, como parte de la Colectiva Editorial Hermanas en la Sombra, la opción que hemos tomado ha sido acompañar los procesos de autorrepresentación mediante la escritura de sus propios libros, donde ellas experimentan con varias estrategias textuales.

\footnotetext{
7 En otros escritos he discurrido sobre los dilemas y las limitaciones de los peritajes antropológicos (Hernández Castillo, 2018), sin embargo, es importante reconocer que la elaboración de peritajes antropológicos o affidavits es una forma de contribuir a las luchas por la justicia de las actoras y los actores sociales con quienes trabajamos. La divulgación de sus luchas o la denuncia de las violencias que afectan sus vidas, a través de la prensa, la radio o materiales audiovisuales, también ha sido otra estrategia de colaboración fundamental en los proyectos antes descritos. Véase <http: / / www.rosalva aidahernandez.com/es/multimedia/>.
} 
Después de 12 años de caminar juntas, hemos aprendido a trabajar con el dolor propio y ajeno, creando espacios de escucha, recurriendo a formas de sanación tradicional, por ejemplo el temazcal, que hemos utilizado como espacio de curación física y espiritual con las integrantes de la Colectiva que ya están fuera. Sabemos que las emociones que cargamos en el cuerpo, si bien son un estímulo que nos moviliza, también nos pueden paralizar y enfermar; necesitamos gestionar ese dolor fuera de las teorizaciones de la academia, desde un lenguaje amoroso que nos permita sanarnos para continuar construyendo comunidad.

En otras experiencias de investigación colaborativa con el equipo de antropología jurídica del CIESAS, hemos realizado talleres de sanación, con el propósito de contribuir a paliar los efectos que la violencia ha tenido en el cuerpo y la mente de las mujeres indígenas con las que trabajamos (véase Hernández Castillo y Terven, 2017). Si bien partimos de una preocupación ética por no revictimizar a través de la recopilación de testimonios, estuvimos conscientes de que, en muchos casos, verbalizar, nombrar los hechos, siempre y cuando sea en el momento propicio y en las condiciones adecuadas, es parte del acomodo y la resignificación del dolor y el trauma en el pasado. Sin embargo, los talleres de sanación nos mostraron que la verbalización no era suficiente, pues el cuerpo guarda también las memorias de dolor y manifiesta conocimientos.

\section{La importancia analítica de reconstruir el vínculo entre violencias estructurales $y$ violencias extremas}

En el nivel teórico y analítico, otra pista importante ha sido llevar el análisis más allá del agravio inmediato y poder contextualizar las violencias estructurales, patriarcales, clasistas y racistas que posibilitan las violencias y la impunidad. En nuestros peritajes argumentamos que el "contexto" es parte del "agravio", y que regresar a la víctima a la situación previa al agravio (como establecen algunas estrategias de reparación en la justicia internacional) implicaría regresarla a los contextos de violencia y vulnerabilidad que en principio hicieron viable la desaparición, el feminicidio o la masacre. La elaboración de peritajes para reparaciones integrales, desde esta perspectiva, conlleva pensar de manera creativa qué tipo de medidas podemos recomendar para que trasciendan la reparación monetaria o el apoyo emocional, y que entrañen medidas de transformación del contexto que facilitó la violencia.

Asimismo, en el plano analítico y político resulta indispensable reconstruir los vínculos entre las violencias extremas que se viven en nuestro país y las economías y culturas militaristas de muerte que circulan de norte a sur. Nuestros trabajos académicos sobre las violencias en México transitan en circuitos internacionales, y pueden contribuir a construir

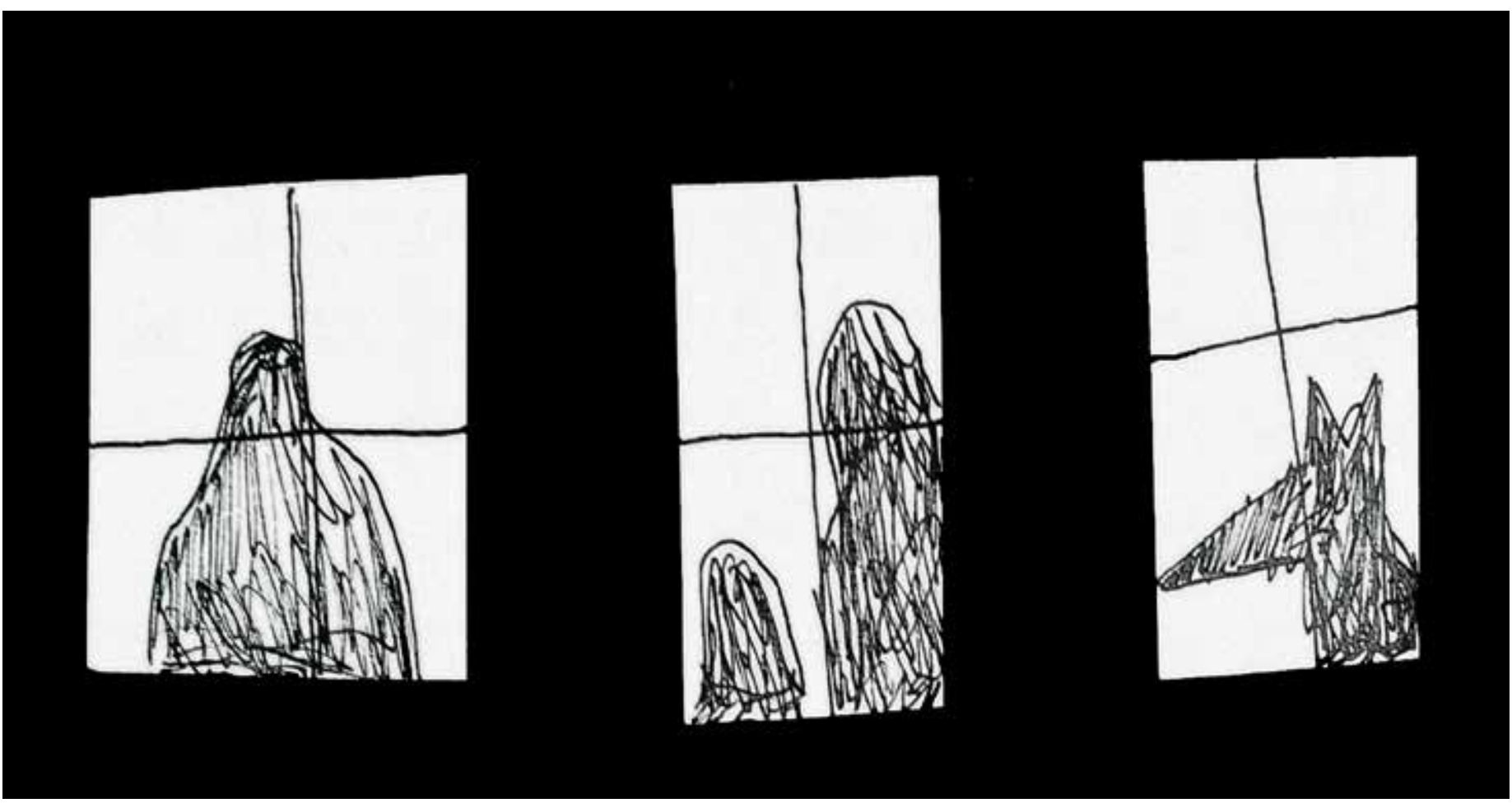


el imaginario de un contexto de "anomia social" producto de instituciones débiles, y de un "atraso en la cultura ciudadana”. En este sentido, para mí ha sido relevante enfatizar en mis trabajos que las violencias que vivimos se dan en un contexto de "cultura de la violencia y militarismo" que se ha globalizado, junto con las armas que la hacen posible.

\section{Reflexiones finales}

Sin proponérmelo, la escritura de este texto me ha permitido hacer un recorrido casi cronológico por mis propias búsquedas metodológicas y políticas, no con el fin de trazar "modelos de investigación de las violencias", sino para compartir los aprendizajes de más de 30 años de investigación feminista. Este artículo no pretende dar respuestas a los retos que supone analizar las múltiples violencias: estructurales, cotidianas, extremas, que están destruyendo el tejido social de nuestras comunidades; antes bien, busca plantear interrogantes y ofrecer algunas pistas para descolonizar nuestra práctica investigativa desde metodologías que reconozcan otros saberes y otras estrategias comunitarias para enfrentar dichas violencias.

Desde mi perspectiva, el dilema no está en elegir entre una antropología del sujeto sufriente (suffering subject) o una antropología del bien (of the good), como señala Joel Robbins (2013), sino en lograr dar cuenta de la complejidad de las violencias y de su impacto en el sufrimiento humano, documentando y examinando a la vez las prácticas de resistencia y resiliencia que los sujetos sociales desarrollan, aun en los contextos de violencias más extremas. Como he apuntado a lo largo de este ensayo, la documentación y el análisis de dichas violencias sólo tienen sentido como parte de esfuerzos colectivos más amplios para confrontarlas y aportar a la construcción de una vida digna en sociedades más equitativas.

Si bien este texto está escrito en primera persona, reconociendo la importancia de situar mi conocimiento
(Haraway, 1991), y hacer evidentes las alianzas políticas a partir de las cuales he elaborado mis problemas de investigación, muchas de las reflexiones que aquí presento son producto de un caminar con distintos colectivos de mujeres. Desde mi formación temprana como feminista en Chiapas aprendí a negociar diferencias como parte del Grupo de Mujeres de San Cristóbal de Las Casas y como voluntaria en su Centro de Apoyo a Mujeres y Menores a principios de los noventa, ${ }^{8}$ donde a través de mi labor con el equipo legal y pedagógico empecé a cuestionar los límites de la justicia estatal para confrontar la violencia de género.

El equipo de antropología jurídica del CIESAS ha sido no sólo mi comunidad académica sino también emocional, y durante 25 años hemos podido crear un espacio de producción de conocimiento y docencia, a contracorriente de una academia positivista que incentiva la competencia y el trabajo individual. A partir de este espacio hemos construido alianzas con organizaciones indígenas, de derechos humanos, de familiares de desaparecidos, con quienes hemos coproducido conocimiento, aprendiendo de sus teorizaciones y sus reflexiones críticas sobre las violencias y las justicias. ${ }^{9}$

Como parte de la Red de Feminismos Descoloniales, desde hace 12 años hemos trabajado de manera colectiva con el objetivo de abonar a la construcción de teorías y prácticas feministas que reconozcan otras formas de ser y estar en el mundo, así como la existencia de otras epistemologías y ontologías, que confrontan las perspectivas universalizantes y etnocéntricas sobre los "derechos de las mujeres". ${ }^{10}$

La Colectiva Editorial Hermanas en la Sombra, en la que confluimos activistas feministas con mujeres que han sufrido la violencia de la prisionización, se ha convertido en un espacio de producción de conocimientos -fuera de los circuitos académicos-, donde la escritura creativa es un instrumento para denunciar las múltiples violencias que afectan las vidas de las mujeres. La escritura es también una forma de repensarnos y reinventarnos como mujeres-desaprendiendo el deber serfemenino que nos inculcaron desde niñas-,

8 Este grupo se fragmentó y dio lugar a varios colectivos más, como la Colectiva Feminista Mercedes Olivera (Cofemo), Colem (libre, en idioma tsotsil), el Observatorio de Muerte Materna de Chiapas, entre otros. En esta época, los diálogos con Guadalupe Cárdenas Zitle, Graciela Freyermuth, Anna María Garza Caligaris, Sebastiana Vázquez Gómez, Juana María Ruiz, Olivia Velázquez Torres, Brenda Velázquez Díaz y Luz María García Moya, fueron fundamentales en mi formación política.

9 En este equipo participamos María Teresa Sierra, Rachel Sieder, Mariana Mora, Dolores Figueroa y, más recientemente, Emiliana Cruz y Carolina Robledo. Natalia De Marinis y Victoria Chenaut, desde el ciEsAs-Golfo, han sido también parte de esta colectiva académica. Véase <http://www.rosalvaaidahernandez.com/es/proyectos-colectivos/ciesas/>. El espacio de nuestra comunidad académica se ha ampliado más allá de las fronteras, formando la Colectiva SOS Anti-Racismo, Género y Justicia, donde participamos todo el equipo del cIEsAs, y nuestras colegas y amigas Shannon Speed, Margo Tamez, Irma Alicia Velásquez Nimatuj y Lynn Stephen.

${ }^{10}$ En la Red de Feminismos Descoloniales participamos Sylvia Marcos, Margara Millán, Gisela Espinosa, Guiomar Rovira, Mariana Mora, Meztli Yoalli Rodríguez, Mariana Favela, Verónica López Nájera, Raquel Gutiérrez y Oscar González. Véase <http: / /www.rosalvaaidahernandez.com/es / proyectos-colectivos/red-feminismos-descoloniales / >. 
y una estrategia para sanar las violencias físicas y emocionales que muchas de nosotras compartimos. Mis reflexiones sobre la escritura y la necesidad de desarrollar otras estrategias textuales para hacer etnografía feminista surgen en diálogo con esta colectiva de mujeres sabias. ${ }^{11}$

En los últimos tres años tuve el privilegio de conocer a distintos colectivos de familiares de desaparecidos como parte del GIASF. Esta colaboración temporal me acercó a un área de especialización totalmente nueva para mí: las ciencias forenses, lo que me llevó a aprender nuevos lenguajes y adquirir nuevos lentes analíticos para ver la realidad. Pero fueron sobre todo las mujeres buscadoras, con su cuidado hacia las personas muertas, su apuesta por la reconstrucción del tejido social, y su modo de repensar la justicia y el resarcimiento, las que nos formaron en una antropología forense desde la ética del cuidado. ${ }^{12}$

La producción del conocimiento en colectivo entraña muchos retos, negociar perspectivas políticas y epistémicas, personalidades y estilos de trabajo, pero sigue siendo una apuesta fundamental para enfrentar los desafíos que implica no sólo hacer etnografía de las violencias, sino vivir en ambientes donde la pedagogía del terror se manifiesta de manera cotidiana.

Quisiera cerrar reconociendo el contexto de nuevas vulnerabilidades en el cual elaboro este texto, en el marco de la pandemia de CoviD-19. Después de un año de cuarentena, en lo que algunos han calificado como la peor crisis sanitaria mundial de los últimos siglos, escribir sobre violencias y la necesidad de construir comunidades emocionales toma una nueva dimensión. Hay quienes han planteado que estamos ante el fin de la antropología fundamentada en el trabajo de campo de larga duración, pues las medidas de distanciamiento social hacen imposible la etnografía presencial, sin poner en riesgo a las personas con quienes trabajamos. ${ }^{13}$
Esto nos ha obligado a recurrir a los expertos en etnografías digitales, para repensar cómo podemos acercarnos a los espacios sociales y a la vida cotidiana de los colectivos con los que nos vinculamos, con las nuevas tecnologías para hacer entrevistas, talleres, grupos focales, así como para documentar, mediante las redes sociales, los espacios digitales en los que las personas con quienes trabajamos se relacionan. ${ }^{14}$ Las limitaciones de estas estrategias son muchas, sobre todo en lo que respecta a aquellas comunidades sin acceso al mundo digital. Se están creando nuevas fronteras entre los mundos interconectados y aquellos que habitan del otro lado de la conectividad.

Sin dejar de reconocer el poder y el control de las grandes empresas de alta tecnología sobre el mundo digital, es importante recordar que se trata de instrumentos que pueden ser empleados también para construir espacios de debate y reflexión crítica y comunidades transnacionales de resistencia. En lo tocante a las comunidades emocionales que hemos construido para trabajar contra la violencia penitenciaria, la pandemia nos ha forzado a establecer alianzas transfronterizas con otras colectivas feministas en América Latina que se ocupan de mujeres en reclusión, para lanzar una campaña latinoamericana de excarcelación de mujeres y grupos vulnerables, cuyas vidas están en riesgo por la covid- $19 .{ }^{15}$ La organización de foros virtuales sobre el tema de la coviD-19 y su impacto en las comunidades indígenas, en las personas en reclusión, en los centros de detención migratoria, ha permitido unir voces $\mathrm{y}$ experiencias de distintos países de América Latina, y en especial pensar juntas y juntos en estrategias y alianzas para enfrentar el nuevo contexto de crisis social y sanitaria.

Es difícil prever el futuro de la etnografía feminista en entornos en los que el distanciamiento social sigue siendo una de las pocas medidas preventivas que funcionan ante la falta de aplicación de una vacuna

${ }^{11}$ La Colectiva Editorial Hermanas en la Sombra está liderada por la poeta Elena de Hoyos, y participamos activamente fuera de la prisión Marina Ruiz, María Vinós, Carolina Corral, Daniela Mondragón, Marcia Trejo, Lucia Espinoza, Manon Vázquez, Valentina Castro, Marisol Hernández del Águila, Suzuki Lee Camacho y Florencia Noble. Dentro de la prisión de Atlacholoaya, Morelos, las integrantes más activas son Elena Basave, Luisa Villanueva, Emelia Chino, Mari Cruz Uribe, Alejandra Apodaca y Nora Guzmán. Véase <http: / /www.rosalvaaidahernandez.com/es/proyectos-colectivos/hermanassombra/>.

${ }^{12}$ El GIASF fue formado por la socióloga Carolina Robledo Silvestre y en él participan Liliana López, May Ek Querales, Sandra Odeth Gerardo, Alejandra Paola Rodríguez, y en el tiempo en que yo fui parte activa del equipo también estuvieron el arqueólogo Alejandro Arteaga y la antropóloga física Albertina Ortega. Véase <http://www.giasf.org/>.

${ }^{13}$ El Colegio de Etnólogos y Antropólogos Sociales en México ha organizado durante la pandemia una serie de webinarios para discutir los retos que enfrenta la antropología a raíz de esta crisis sanitaria. Estos debates se pueden ver en su canal de YouTube <https: / /www.youtube.com/watch?v=onRiebuscNs>.

${ }^{14}$ En el CIESAS se ha impulsado una serie de conferencias con especialistas en etnografías digitales, con el título de Antropología del confinamiento. Véase <https://www.ciesas.edu.mx/la-antropologia-en-confinamiento/>

${ }^{15}$ Esta campaña ha sido lanzada por la Red Anticarcelaria de Abya Yala, a partir de un catastro de organizaciones que trabajan en espacios de reclusión realizado por la Colectiva Hermanas en la Sombra y la organización Pájarx entre Púas de Chile. Véase <https: / / conlaa.com/liberarlas-es-justicia-el-covid-y-la-crisis-humanitaria-en-las-prisiones-de-america-latina/>. 
a toda la población. Sin embargo con las nuevas tecnologías digitales y los medios de comunicación más tradicionales, la capacidad de seguir construyendo comunidades emocionales, políticas y de resistencia se ha probado efectiva durante estos meses de cuarentena. Como señaló la escritora feminista india Arundhati Roy (2020), "la pandemia es un portal": lo que encontremos del otro lado dependerá de nuestra creatividad, de la habilidad política de construir alianzas, y de la capacidad de producir pensamiento crítico que contribuya a las luchas por la justicia social.

\section{Fuentes}

AnZaldúa, Gloria

1987 Borderlands/La Frontera. The New Mestiza, Aunt Lute Books, San Francisco.

Aranguren Romero, Juan Pablo

2016 Cuerpos al límite: tortura, subjetividad y memoria en Colombia (1977-1982), Universidad de los Andes-Facultad de Ciencias Sociales, Bogotá.

BEHAR, RUTH

2003 Translated Woman: Crossing the Border with Esperanza's Story, Beacon Press, Boston.

BEHAR, RUTH

y Deborah A. Gordon (eds.)

1995 Women, Writing Culture, University of California Press, Berkeley.

Belausteguigoitia Rius, Marisa

y María Josefina SAldaña-Portillo (COORDS.)

2015 Des/posesión: género, territorio y luchas por la autodeterminación, Universidad Nacional Autónoma de México-Programa Universitario de Estudios de Género, México.

Bell, Diane, Pat Caplan

Y JAHAN KARIM WAZIR (EDS.)

1993 Gendered Fields: Women, Men, and Ethnography, Routledge, Londres y Nueva York.

Berry, Maya J., Claudia Chávez Argüelles,

Shanya Cordis, SARAH IHMOUd

y Elizabeth Velásguez Estrada

2017 "Toward a fugitive anthropology: Gender, race, and violence in the field", en Cultural Anthropology, vol. 32, núm. 4, noviembre, pp. 537-565.

Cacho, Lisa Marie

2012 Social Death: Racialized Rightlessness and the Criminalization of the Unprotected, New York University Press, Nueva York.

Corral, Carolina

2016 Semillas de Guamúchil, Instituto Mexicano de Cinematografía, México <https: / /vimeo.com/

Crenshaw, Kimberlé W.

1991 "Mapping the margins: Intersectionality, identity politics, and violence against women of color", en Stanford Law Review, vol. 43, núm. 6, pp. 1241-1299.

Das, Veena

2008 Veena Das: sujetos del dolor, agentes de dignidad, editado por Francisco A. Ortega, Pontificia Universidad Javeriana-Instituto Pensar/ Universidad Nacional de Colombia, Bogotá.
De Marinis, Natalia

2017 "Etnografiar (en) el terror: el 'ser testigo' y la construcción de comunidades político-afectivas. Reflexiones a partir de una experiencia de campo", en Yerko Castro Neira y Adèle Blazquez (coords.), Micropolíticas de la violencia. Reflexiones sobre el trabajo de campo en contextos de guerra, conflicto y violencia, Laboratorio Mixto Internacional (Cuadernos de Trabajo de MESO, 5/2017), México, pp. 9-21.

Dreyfus, JEAN-MARC

y ÉlisABeth Anstett (EDs.)

2015 Human Remains and Mass Violence: Methodological Approaches, Manchester University Press, Manchester.

FERrándiz, Francisco

2014 El pasado bajo tierra: exhumaciones contemporáneas de la Guerra Civil, Anthropos, Barcelona.

García González, Andrea

2019 "Desde el conflicto: epistemología y política en las etnografías feministas", en Antípoda. Revista de Antropología y Arqueología, vol. 35, núm. 1, pp. 3-21.

García-Santesmases Fernández, Andrea

2019 "Evocando deseos y revolviendo malestares: la im-pertinencia de las emociones en mi trabajo etnográfico", en Antípoda. Revista de Antropología y Arqueología, vol. 35, núm. 1, pp. 69-89.

Gordon, EDMUnd T.

2007 "The Austin School Manifesto: An approach to the Black or African diaspora”, en Cultural Dynamics, vol. 19, núm. 1, pp. 93-97.

Hale, Charles

2008 "Introduction", en Charles Hale (ed.), Engaging Contradictions: Theory, Politics, and Methods of Activist Scholarship, University of California Press, Berkeley, pp. 1-29.

Haraway, Donna Jeanne

1991 "Situated knowledges: The science question in feminism and the privilege of partial perspective", en Simians, Cyborgs, and Women: The Reinvention of Nature, Routledge, Nueva York, pp. 183-203.

Hernández Castillo, Rosalva Aída

2001a "Entre el etnocentrismo feminista y el esencialismo étnico: las mujeres indígenas y sus demandas de género", en Debate Feminista, vol. 24, núm. 1, pp. 206-229.

Hernández Castillo, Rosalva Aída

2001b Histories and Stories from Chiapas: Border Identities in Southern Mexico, University of Texas Press, Austin.

Hernández Castillo, Rosalva Aída

2001c La otra frontera: identidades múltiples en el Chiapas poscolonial, Centro de Investigaciones y Estudios Superiores en Antropología Social/ Miguel Ángel Porrúa, México.

Hernández Castillo, Rosalva Aída

2013 ¿Del Estado multicultural al Estado penal? Mujeres indígenas presas y criminalización de la pobreza en México", en María Teresa Sierra Camacho, Rosalva Aída Hernández Castillo y Rachel Sieder (eds.), Justicias indígenas y Estado. Violencias contemporáneas, Facultad Latinoamericana de Ciencias Sociales/Centro de Investigaciones y Estudios 
Superiores en Antropología Social, México, pp. 299-338.

Hernández Castillo, Rosalva Aída

2015 "Hacia una antropología socialmente comprometida desde una perspectiva dialógica y feminista", en Xochitl Leyva Solano (ed.), Prácticas otras de conocimiento(s): entre crisis, entre guerras, tomo II, Cooperativa Editorial RETOS, San Cristóbal de Las Casas, pp. 83 106.

Hernández Castillo, Rosalva Aída

2016a "Feminist activist research and intercultural dialogues", en June C. Nash y Hans C. Buechler (eds.), Ethnographic Collaborations in Latin America: The Effects of Globalization, Palgrave Macmillan, Nueva York, pp. 25-47.

Hernández Castillo, Rosalva Aída

2016b Multiple Injustices: Indigenous Women, Law, and Political Struggle in Latin America, The University of Arizona Press, Tucson.

Hernández Castillo, Rosalva Aída (coord.)

2017 Resistencias penitenciarias: investigación activista en espacios de reclusión, Grupo Internacional de Trabajo Sobre Asuntos Indígenas / Colectiva Editorial Hermanas en la Sombra/ Juan Pablos Editor, México.

Hernández Castillo, Rosalva Aída

2018 "Retos y posibilidades de los peritajes antropológicos: reflexiones desde la experiencia mexicana", en Abya-Yala: Revista sobre Acesso à Justiça e Direitos nas Américas, vol. 2, núm. 2, pp. 57-85.

Hernández Castillo, Rosalva Aída

2019a "La antropología jurídica feminista y sus aportes al trabajo forense con familiares de desaparecidos: alianzas y colaboraciones con 'Las Rastreadoras de El Fuerte'", en AbyaYala: Revista sobre Acesso à Justiça e Direitos nas Américas, vol. 3, núm. 2, pp. 94-119.

Hernández Castillo, Rosalva Aída

$2019 \mathrm{~b}$ "Racialized geographies and the "War on Drugs': Gender violence, militarization, and criminalization of Indigenous peoples", en The Journal of Latin American and Caribbean Anthropology, vol. 24, núm. 3, pp. 635-652.

Hernández Castillo, Rosalva Aída

2021 "Prison as a Colonial enclave: Incarcerated Indigenous women resisting multiple violence", en Lynn Stephen y Shannon Speed (eds.), Indigenous Women and Violence: Feminist Activist Research in Heightened States of Injustice, The University of Arizona Press, Tucson, pp. 43-73.

Hernández Castillo, Rosalva Aída

y Gisela Espinosa Damián

2012 "New Political actors in Rural Mexico: The challenges and achievements of Peasant and Indigenous women", en K. Z. Dellacioppa y C. Weber (eds.), Cultural Politics and Resistance in the 21st Century: Community-Based Social Movements and Global Change in the Americas, Palgrave Macmillan, Nueva York, pp. 23-48.

Hernández Castillo, Rosalva Aída

y Carolina Robledo Silvestre

2020 Nadie detiene al amor. Historias de vida de familiares de personas desaparecidas en el norte de Sinaloa, Universidad Nacional Autónoma de México-Instituto de Investigaciones Jurídicas / Colectiva Editorial Hermanas en la Som-
bra/Grupo de Investigación en Antropología Social y Forense/Desaparecidos de El Fuerte y Zona Norte/Fundar / Documenta, México.

Hernández Castillo, Rosalva Aída

Y ADRIANA TERVEN

2017 "Rutas metodológicas: hacia una antropología jurídica crítica y colaborativa”, en Rachel Sieder (coord.), Exigiendo justicia y seguridad: mujeres indígenas y pluralidades legales en América Latina, Centro de Investigaciones y Estudios Superiores en Antropología Social (Publicaciones de la Casa Chata), México, pp. 485-524.

HOOKS, BELL

2000 All about Love: New Visions, William Morrow, Nueva York.

JiMENO, MYRIAM

2000 "La emergencia del investigador ciudadano: estilos de antropología y crisis de modelos en la antropología colombiana", en Jairo Tocancipá (ed.), La formación del Estado-nación y las disciplinas sociales en Colombia, Universidad del Cauca-Departamento de Antropología-Facultad de Ciencias Sociales, Popayán, pp. 157-190.

JIMENO, MYRIAM

2010 "Emociones y política. La 'víctima' y la construcción de comunidades emocionales", en Mana: Estudos de Antropologia Social, vol. 16, núm. 1, pp. 99-121.

Kleinman, Arthur

Y JOAN KLEINMAN

1996 "The appeal of experience; the dismay of images: Cultural appropriations of suffering in our times", en Daedalus, vol. 125, núm. 1, pp. 1-24.

LORDE, Audre

1984 Sister Outsider: Essays and Speeches, Crossing Press/Trumansburg, Nueva York.

MACLEOD, MORNA

y Natalia De Marinis

2019 Comunidades emocionales. Resistiendo a las violencias en América Latina, Instituto Colombiano de Antropología e Historia, Bogotá.

Mohanty, Chandra TAlpade

1984 "Under Western eyes: Feminist scholarship and colonial discourses", en On Humanism and the University I: The Discourse of Humanism, vol. 12, núm. 3, pp. 333-358.

Moore, Henrietta L.

2009 Antropología y feminismo, Cátedra/Universidad de Valencia/Instituto de la Mujer, Madrid.

REINHARZ, SHULAMIT

Y LYNN DAVIDMAN

1992 Feminist Methods in Social Research, Oxford University Press, Nueva York.

Rivera, Mariana

2018 El hilo de la memoria, Urdimbre Audiovisual, México <https: / / vimeo.com/297415375> [ 15 de noviembre de 2021 ].

RoBbins, JoEL

2013 "Beyond the suffering subject: Toward an anthropology of the good", en Journal of the Royal Anthropological Institute, vol. 19, núm. 3 , septiembre, pp. 447-462.

RoJAs-PEREZ, IsAiAs

2017 Mourning Remains: State Atrocity, Exhumations, and Governing the Disappeared in Peru's 
Postwar Andes, Stanford University Press, Stanford.

Roy, ARundhati

2020 "La pandemia es un portal", en La Jornada, 13 de abril <https://www.jornada.com.mx/ ultimas / mundo/2020/04/13/la-pandemia-

Ruiz TRejo, Marisa es-un-portal-9285.html>[5 deagostode 2020].

2016

"Historia de las epistemólogas, metodólogas y feministas en México y Centroamérica", ponencia presentada en el III Congreso de Historia Intelectual de América Latina "Formas de historia intelectual. Teoría y praxis", El Cole-

SAID, EDWARD W. gio de México, México.

1978 Orientalism, Routledge \& Kegan, Londres.

SANFORD, VICTORIA

2003 Buried Secrets: Truth and Human Rights in Guatemala, Palgrave Macmillan, Nueva York.

Scheper-Hughes, Nancy

1983 "The problem of bias in androcentric and feminist anthropology", en Women's Studies, vol. 10, núm. 2, pp. 109-116.

Segato, Rita Laura

2013 La escritura en el cuerpo de las mujeres asesinadas en Ciudad Juárez: territorio, soberanía y crímenes de segundo estado, Tinta Limón

Smith, Christen A. Ediciones, Buenos Aires.

2016 "Facing the Dragon; Black mothering, sequelae and Gendered Necropolitics in the Ameri- cas”, en Transforming Anthropology, vol. 24, núm. 1, pp. 1-94.

Sierra Camacho, María Teresa,

Rosalva Aída Hernández Castillo

y RACHEL Sieder (EDS.)

2013 Justicias indígenas y Estado. Violencias contemporáneas, Facultad Latinoamericana de Ciencias Sociales/Centro de Investigaciones y Estudios Superiores en Antropología Social, México.

Speed, Shannon

2008 "Forged in dialogue: Toward a critically engaged activist research", en Charles Hale (ed.), Engaging Contradictions: Theory, Politics, and Methods of Activist Scholarship, University of California Press, Berkeley, pp. 213-235.

SuÁREZ-NAVAZ, LiLIANA

2006 Rebordering the Mediterranean: Boundaries and Citizenship in Southern Europe, Berghahn Books, Nueva York.

TAMEZ, MARGO

2007 Raven Eye, The University of Arizona Press, Tucson.

VALENCIA, SAYAK

2010 Capitalismo gore, Melusina, Barcelona.

Velásguez NimatuJ, Irma Alicia

2018 Lunas y calendarios, Editorial Cultura, Guatemala.

Wolf, Diane L. (ED.)

1996 Feminist Dilemmas in Fieldwork, Westview Press, Boulder. 